\title{
A chemical approach to "rewire" neural progenitor cells
}

\author{
Cell Research (2014) 24:641-642. doi:10.1038/cr.2014.51; published online 18 April 2014
}

Generation of neural progenitor cells (NPCs) from pluripotent stem cells including ESCs and iPSCs and derivation of NPCs from somatic tissues have been considered promising approaches that could be used therapeutically to restore function in patients suffering neurodegenerative diseases. A new study published in Cell Research shows, for the first time, the generation of NPCs from somatic cells by small molecule compounds under hypoxia without exogenous transcription factors.

Neural progenitor cells (NPCs) are multipotent progenitor cells that possess the ability of self-renewal and multi-lineage neural differentiation [1]. Increasing attention has been placed on NPCs due to their potential as a source of replacement cells for treating neurodegenerative diseases. Steady progress from previous animal studies has shown the potential of NPC transplantation in preserving or restoring brain function, which fuels efforts to establish stemcell-based transplantation therapies in humans [2]. The first FDA-approved stem cell trial for amyotrophic lateral sclerosis (ALS) has shown that transplanted human fetal NPCs dramatically slow down the progression of the disease in a successful Phase 1 trial, which paves the way for further applications of NPCs in clinical treatment of neurodegenerative diseases.

NPCs exist in small numbers in inaccessible niches in the body, and therefore it is not feasible to harvest NPCs for autologous transplantation. As an alternative to allogeneic transplantation of fetal NPCs, strategies have been developed to generate autologous NPCs from other cell types. Converting somatic cells into pluripotent stem cells, also known as reprogramming, through the enforced expression of four factors Oct3/4, Sox2, Klf4 and c-Myc, was first introduced by Yamanaka and colleagues in 2006 [3]. This discovery opened a new era in regenerative medicine and provided a novel opportunity to generate an unlimited number of NPCs from induced pluripotent stem cells (iPSCs) in vitro for transplantation purposes.

Recently, iPSC-derived NPCs have been successfully generated [4]; however, clinical applications have been limited due to the risk of tumor formation resulting from incomplete differentiation of pluripotent cells. Somatic cells, including fibroblasts and hematopoietic stem cells, have been directly converted to expandable NPCs by overexpression of exogenous transcription factors [5, 6], which could circumvent the risk associated with iPSCs. However, similar to iPSC technology, direct conversion of somatic cells to NPCs by introducing exogenous factors raises clinical safety concerns. Therefore, the development of an approach to induce NPCs from somatic cells using only small molecule compounds offers a potentially safer strategy to generate patient-specific NPCs for treating neurological diseases.

To achieve this goal, Cheng et al. [7] identified a chemical cocktail that induces NPCs from terminally differentiated cells under physiological hypoxic condition. It was hypothesized that chemicals known to promote reprogramming could facilitate the cells to enter into a "plastic" state, which could allow them to be converted to NPCs under neural-specific conditions.
Emphasis was additionally given to compounds that upregulate Sox2 $[8,9]$ due to its key role in NPCs. Given that hypoxia represents a key feature of in vivo stem cell niches and facilitates cellular reprogramming, the authors included physiological hypoxia as one of the test parameters. The combination of Valproic acid (VPA, HDAC inhibitor), $\underline{\mathrm{CHIR}} 99021$ (GSK-3 $\beta$ inhibitor) and Repsox (TGF- $\beta$ inhibitor), hereafter referred to as VCR, was found to induce the transition of MEFs to compact cell colonies that are positive for alkaline phosphatase activity (intermediate "plastic" cells) under hypoxic conditions $\left(5 \% \mathrm{O}_{2}\right)$. This phenomenon was not observed under normoxic conditions, suggesting the critical role of hypoxia in VCR-induced intermediary transition. Moreover, through the use of lineage-specific culture conditions accompanied by multiple rounds of neurosphere suspension culture, a homogenous NPC population, referred to as chemical-induced NPC (ciNPC), was obtained from the intermediate cells. Further characterization showed a high degree of similarity between ciNPCs and mouse brain-derived NPCs in their proliferation and self-renewal abilities, transcription profiles, as well as their multipotency in vitro and in vivo. Intriguingly, VCR could be replaced by alternative inhibitors targeting the HDACs, GSK-3 and TGF- $\beta$ kinase pathways, indicating a central role of these pathways in the direct conversion of MEFs into NPCs. In addition, this study demonstrated the feasibility of generating ciNPCs from somatic cells at a later developmental stage (i.e., neonatal tail-tip fibroblasts) or from human 
cells, suggesting a broad application potential of this strategy in both basic research and clinical settings. Thus, this study provides a novel strategy to directly obtain potentially safe NPCs from various types of somatic cells.

Generation of NPCs through iPSC differentiation or lineage-specific conversion of somatic cells has major implications for regenerative medicine. As discussed above, these re-derived autologous NPCs could be an important alternative to fetal spinal cord-derived allogeneic NPCs for treating neurodegenerative diseases. In addition to therapeutic purposes, disease modeling based on patient-derived NPCs and neural cells could yield insights into the mechanisms underlying neurodegenerative diseases such as Parkinson's disease, Alzheimer's disease and ALS. Although protocols have been developed to generate NPCs from both routes, safety concerns from the introduction of exogenous genes remain a major roadblock against clinical translation of these technologies; continued expression of exogenous factors in NPCs may affect their function and thus hinder disease modeling. For the first time, Cheng and colleagues introduce a novel approach to obtain NPCs using only chemical cocktails and physiological hypoxia. Their results showed that conversion towards a neural fate by inhibition of HDACs, TGF- $\beta$ and GSK-3 is accompanied by the activation of Sox2, which is consistent with previous findings showing that Sox2 alone can convert somatic cells into NPCs $[5,10]$. However, the mechanistic link between these pathways and a Sox2-driven neural conversion program is not explored in the present study. Studying the mechanisms of chemically induced NPC conversion may result in more direct and efficient methods in the future. NPCs reside in hypoxic niches of the central nervous system where their proliferation and differentiation are influenced by hypoxia. Accumulating evidence has shown that hypoxia facilitates cellular reprogramming and significantly enhances the self-renewal and neurogenic abilities of NPCs [11]. It is therefore not surprising that physiological hypoxia could promote the generation of NPCs directly from somatic cells, though the pathways through which hypoxia mediates ciNPC conversion need to be further elucidated. Once these pathways are identified, it may be possible to substitute hypoxia with new compounds targeting them. It would be interesting to test whether the chemical cocktails could facilitate lineage conversion or regeneration in vivo. In this scenario, the cells will be naturally under physiological hypoxic conditions. Obviously, serial neurosphere formation is not possible in vivo and therefore new cocktails that enable more direct and efficient ciNPC conversion may be required to achieve this. In any case, long-term in vivo follow-up studies are necessary to rigorously test the safety of ciNPCs. The current study, following the report of chemically induced pluripotent stem cells [12], signifies a new phase of cell fate manipulation that is opening up exciting possibilities for regenerative medicine.

Min-Zu Wu ${ }^{1}, \mathrm{Mo} \mathrm{Li}^{1}$, Guang-Hui $\mathrm{Liu}^{2,3}$, Juan Carlos Izpisua Belmonte ${ }^{1}$

${ }^{I}$ Gene Expression Laboratory, Salk Institute for Biological Studies, 10010 N. Torrey Pines Rd., La Jolla, CA 92037, USA; ${ }^{2}$ National Laboratory of Biomacromolecules, Institute of Biophysics, Chinese Academy of Sciences, Beijing 100101, China; ${ }^{3}$ Beijing Institute for Brain Disorders, Beijing 100875, China

Correspondence: Juan Carlos Izpisua Belmonte E-mail: belmonte@salk.edu

\section{References}

1 Gage FH, Kempermann G, Palmer TD, et al. J Neurobiol 1998; 36:249-266.

2 Lindvall O, Kokaia Z. Nature 2006; 441:1094-1096.

3 Takahashi K, Yamanaka S. Cell 2006; 126:663-676.

4 Yamanaka S. Cell 2009; 137:13-17.

5 Giorgetti A, Marchetto MC, Li M, et al. Proc Natl Acad Sci USA 2012; 109:1255612561.

6 Thier M, Wörsdörfer $\mathrm{P}$, Lakes $\mathrm{YB}$, et al. Cell Stem Cell 2012; 10:473-479.

7 Cheng L, Hu W, Qiu B, et al. Cell Res 2014; 24:665-679.

8 Jung DW, Kim WH, Williams DR. ACS Chem Biol 2013; 9:80-95.

9 Ichida JK, Blanchard J, Lam K, et al. Cell Stem Cell 2009; 5:491-503.

10 Ring KL, Tong LM, Balestra ME, et al. Cell Stem Cell 2012; 11:100-109.

11 Simon MC, Keith B. Nat Rev Mol Cell Biol 2008; 9:285-296.

12 Hou P, Li Y, Zhang X, et al. Science 2013; 341:651-654. 